NASA Technical Memorandum 101965

ICOMP-89-6

\title{
The Response of a Laminar Boundary Layer in Supersonic Flow to Small Amplitude Progressive Waves
}

(DASA-TH-101965) THE EESECASE CE A LAHINAE

Peter W. Duck Institute for Computational Mechanics in Propulsion Lewis Research Center Cleveland, Ohio

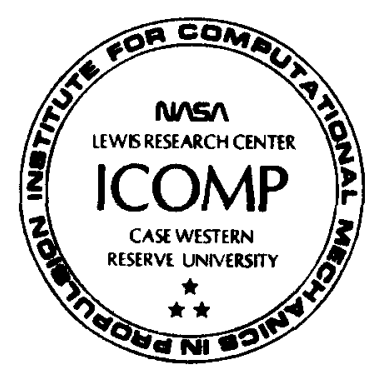


The Response of a Laminar Boundary Layer in Supersonic Flow to

Smal1 Amplitude Progressive Waves

Peter W. Duck*

Institute for Computational Mechanics in Propulsion

Lewis Research Center

Cleveland, Ohio 44135

\section{Abstract}

In this paper the effect of a small amplitude progressive wave on the laminar boundary layer on a semi-infinite flat plate, due to a uniform supersonic freestream flow, is considered.

The perturbation to the flow divides into two streamwise zones. In the first, relatively close to the leading edge of the plate, on a transverse scale comparable to the boundary layer thickness, the perturbation flow is described by form of the unsteady linearised compressible boundary layer equations. In the freestream, this component of flow is governed by the wave equation, the solution of which provides the outer velocity conditions for the boundary layer. This system is solved numerically, and also the asymptotic structure in the far downstream limit is studied. This reveals a breakdown and a subsequent second streamwise zone, where the flow disturbance is predominantly inviscid. The two zones are shown to match in a proper asymptotic sense.

*Work funded under Space Act Agreement C99066G; presently at Department of Mathematics, University of Manchester, Manchester, England. 


\section{Introduction}

Much work has been carried out on the response of a two-dimensional imcompressible laminar boundary layer on semi-infinite flat plate to time-harmonic osclllatory perturbations (particularly those of infinite wavelength in the streamwise direction) of the freestream. Most of the effort has been involved with small amplitude perturbations; included in this category are the works of Lighthill (1954), Lam and Rot t (1960) and Ackerberg and Phillips (1972). Close to the leading edge, the flow is of Blasius type, whilst far downstream the boundary layer takes on a double structure, comprising an inner Stokes-type layer, and an outer Blasius-type layer.

Numerical investigations to this small amplitude problem, describing the flow from the leading edge, to far downstream have been conducted by Ackerberg and Phillips (1970) and Coldstein et al (1983). Some details of the far downstream behaviour of this problem have in the past been the subject of some controversy. At large distances downstream of the leading edge, a set of eigensolutions must exist, which decay exponentially fast downstream in the streamwise direction (this reflects the effect of the particular conditions prevailing upstream). One set of eigensolutions found by Lam and Rott (1960) and by Ackerberg and Phillips (1972) is determined by conditions close to the wall, and has decay rates that decrease as the eigenvalue increases. A second set of eigensolutions has been found by Brown and Stewartson (1973a,b), and these are determined primarily by conditions at the outer edge of the boundary layer, and are characterised by a decay rate that increases with increasing order. Some discussion of this apparent discrepency is to be found in Goldstein et al. (1983).

Further downstream still, Goldstein (1983) showed how the eigensolutions 
of Lam and Rott (1960), which initially decay, develop into the classical, high Reynolds number limit for Tollmien-Schlichting modes (including the unstable mode) for Blasius-type boundary layers. Although the Lam and Rott (1960) eigensolutions decay downstream, they also osclllate with increasingly rapid (spatial) frequency, and far downstream, transverse and streamwise gradients must become comparable, giving rise to an ent irely new structure, in which transverse pressure gradients play a key role.

Returning to the boundary layer problem, non-small oscillations of the freestream (but still limited to non-reversing freestreams) have been tackled by Moore (1951), (1957) and Pedley (1972) for regions close to the leading edge of the plate, and it is again found that the flow is Blasius like. Lin (1956), Gibson (1957) and Pedley (1972) studied the far downstream region, and found the flow takes on a double structure similar to that observed in the analogous small amplitude case.

Duck (1989) has presented numerical results which extend from the leading edge, to far downstream, in this case.

All these aforementioned papers were concerned with purely temporal flow oscillations (i.e. oscillations of infinite streamwise wavelength). The effect of a small amplitude progressive wave on an incompressible boundary layer has been investigated by Kestin, Maeder and Wang (1961) and Patel (1975). The former authors considered the low frequency 1 imit to the problem, for the very particular case when the wavespeed equalled the mean far field velocity. Patel (1975) gave results for both high and low frequency, the latter being obtained using an empirical momentum integral appraoch. A number of experimental results were also presented, and compared with theory.

In this paper we consider the effect of a two-dimensional small 
amplitude progressive wave in supersonic freestream, on a laminar boundary layer on a flat plate. Here the situation is rather different to the incompressible case in a number of respects. Although eigenfunctions similar to those of Lam and Rott (1960) undoubtedly exist, these are not expected to develop into growing Tollmien-Schlichting waves (at least not in the case of two-dimensional flows - see Ryzhov and Zhuk 1980 and Duck 1985). Although planar Tollmien-Schlichting waves are present downstream, these all decay and so are of little consequence. In this paper, as well as presenting results for the boundary layer region, we go on to consider the development of the flow far downstream, which turns out to be predominantly inviscid. A further difference found is that in the case of these supersonic flows (unlike incompressible flows) if the progressive wave is to sat isfy the appropriate governing equations in the farfield, the spatial and temporal wavelengths are linked. (A somewhat related study, of a forced flow but at finite Reynolds numbers has been made by Mack 1975).

The layout of the paper is as follows: in Section 2 we consider the "fore region" in which the flow close to the plate is governed by a form of the compressible boundary layer equations. We look at the downstream limit of this zone, and then in Section 3 we consider the downstream region, wherein transverse variations of pressure become important; the regions studied separately in Sections 2 and 3 are seen to match (in an asymptotic sense). In Section 4 we show how results from the previous two sections correctly match, and finally in section 5 we present a few conclusions. 


\section{The fore region}

We consider the viscous supersonic two-dimensional flow over a semi-infinite, stationary plate. We take the orlgin at the leading edge of the plate, $x^{*}$ the coordinate along the plate, $y^{*}$ the coordinate normal to the plate, and $u^{*}$ and $v^{*}$ the velocity components in the $x^{\star}$ and $y^{\star}$ directions respectively. $t^{*}$ denotes time, $\rho^{\star}$ the fluid density $\mu^{\star}$ the viscosity of the fluid. $p^{\star}$ and $T^{\star}$ are defined to be pressure and temperature in the fluid; $c_{p}\left(c_{v}\right)$ is the specific heat of the fluid at constant pressure (volume), and $\sigma-\mu^{\star} c_{p} / K^{\star}$ is the Prandt 1 number, ( $K^{\star}$ being the coefficient of thermal diffusivity); $c_{p}, c_{v}$ and $\sigma$ are all assumed to be constants. Subcript $\propto$ will be used to denote farfield, unperturbed conditions.

The fluid is also assumed to sat isfy the following equation of state

where $\quad R^{*}=c_{p}-c_{v}$.

$$
P^{\star}=\rho^{\star} \mathbf{R}^{\star} T^{\star}
$$

We consider here, first the fluid in the far field, where the effects of viscosity are expected to be negligible. In this region, we take the flow to comprise aniform steady stream parallel to the plate, perturbed by a small amplitude $(O(\epsilon) \ll 1)$ progressive wave.

We therefore write

$$
\begin{aligned}
& u^{\star}=U_{\infty}^{\star}+\varepsilon u_{1}^{\star}\left(x^{*}, y^{*}, t^{*}\right)+\ldots \\
& v^{\star}-\varepsilon v_{1}^{*}\left(x^{*}, y^{\star}, t^{*}\right)+\ldots \\
& \rho^{\star} \rho_{\infty}^{\star}+\epsilon \rho_{1}^{*}\left(x^{*}, y^{\star}, t^{\star}\right)+\ldots \\
& T^{\star}=T_{\infty}^{*}+\epsilon T_{1}^{*}\left(x^{\star}, y^{\star}, t^{\star}\right)+\ldots \\
& p^{*}=p_{\infty}^{*}+\varepsilon p_{1}^{*}\left(x^{*}, y^{*}, t^{*}\right) \text {. }
\end{aligned}
$$


The leading order equation of state gives

$$
P_{\propto}^{\star}=\rho_{\propto}^{\star} R \mathbf{R T}_{\propto}^{\star}
$$

Substitution of (2.3) into the momentum equations and continuity equation yields (at $O(\varepsilon)$ )

$$
\begin{aligned}
& \rho_{\infty}^{\star}\left[\frac{\partial u_{j}^{\star}}{\partial t^{*}}+u_{\infty}^{\star} \frac{\partial u_{j}^{\star}}{\partial x^{\star}}\right]=-\frac{\partial p_{j}^{\star}}{\partial x^{\star}} \\
& \rho_{\infty}^{\star}\left[\frac{\partial v_{1}^{\star}}{\partial t^{*}}+u_{\infty}^{\star} \frac{\partial v_{1}^{\star}}{\partial x^{\star}}\right]=-\frac{\partial p_{1}^{*}}{\partial y^{*}} \\
& \frac{\partial \rho_{1}^{\star}}{\partial t^{*}}+u_{\propto}^{\star} \frac{\partial \rho_{1}^{\star}}{\partial x^{*}}+\rho_{\propto}^{\star} \frac{\partial u_{1}^{*}}{\partial x^{\star}}+\rho_{\propto}^{\star} \frac{\partial u_{j}^{*}}{\partial y^{*}}=0 .
\end{aligned}
$$

Differentiating (2.5) with respect to $x^{\star},(2.6)$ with respect to $y^{\star}$, and adding yields

$\frac{\partial^{2} p_{1}^{\star}}{\partial x^{\star 2}}+\frac{\partial^{2} p_{1}^{\star}}{\partial y^{\star 2}}=\frac{\partial^{2} \rho_{1}^{\star}}{\partial t^{\star 2}}+2 u_{\infty}^{\star} \frac{\partial^{2} \rho_{1}^{\star}}{\partial x^{\star} \partial t^{\star}}+u_{\infty}^{\star 2} \frac{\partial^{2} \rho_{1}^{\star}}{\partial x^{\star 2}}$

If we write

with

$$
a_{\infty}^{2}=\gamma^{\star} T_{\propto}^{*}
$$

where $\mathbf{a}_{\propto}^{\star}$ denotes the speed of sound in the far field, then (2.8) may be written entirely in terms of $\mathbf{p}_{1}^{*}$, viz

$$
a_{\infty}^{\star 2}\left[\frac{\partial^{2} p_{1}^{\star}}{\partial x^{\star 2}}+\frac{\partial^{2} p_{j}^{\star}}{\partial y^{\star}}\right]-\left[\frac{\partial}{\partial t^{\star}}+u_{\infty}^{\star} \frac{\partial}{\partial x^{\star}}\right]^{2} P_{1}^{\star}
$$

We now seek progressive wave solutions of this equation, writing

$$
p_{1}^{\star}=\hat{p}_{1}^{\star}\left\{\exp \left[i \alpha^{\star}\left(x^{\star}-c^{\star} t^{\star}+\lambda^{\star} y^{\star}\right)\right]+\exp \left[i \alpha^{\star}\left(x^{\star}-c^{\star} t^{\star}-\lambda^{\star} y^{\star}\right)\right]\right\}
$$


(corresponding to an oblique wave, in general), where $\hat{p}_{1}{ }^{\star}$ is a constant. Substitution of (2.12) into (2.11) reveals that we must have

$$
c^{\star}=u_{\infty}^{\star}\left[1 \pm \frac{1}{M_{\infty}}\left(1+\lambda^{\star 2}\right)^{\frac{1}{2}}\right]
$$

where $M_{\infty}$ denotes the unperturbed freestream Mach number, $u_{\infty}^{*} / a_{\infty}^{*}$.

It is now possible to write down the progressive wave solution for $u_{l}^{*}$ and $v_{I}^{*}$, namely

$$
u_{1}^{\star}=\frac{-p_{1}^{\star}}{\left(u_{\alpha}^{\star}-c^{\star}\right) p_{\infty}}\left\{\exp \left[i \alpha^{\star}\left(x^{\star}-c^{\star} t^{\star}+\lambda^{\star} y^{\star}\right)\right]+\exp \left[i \alpha^{\star}\left(x^{\star}-c^{\star} t^{\star}-\lambda^{\star} y^{\star}\right)\right]\right\},
$$

$$
v_{1}^{\star}=-\frac{\lambda p_{1}^{\star}}{\left(t_{\infty}-c^{\star}\right)} \rho_{\rho_{\infty}}^{\star}\left\{\exp \left(i \alpha^{\star}\left(x^{\star}-c^{\star} t^{\star}+\lambda^{\star} y^{\star}\right)-\exp \left[i \alpha^{\star}\left(x^{\star}-c^{\star} t^{\star}-\lambda^{\star} y^{\star}\right)\right]\right\}\right.
$$

Equation (2.14b) also satisfies the normal flow condition, although the no-slip condition is violated by (2.14a), since

$$
u_{1}^{\star}\left(y^{\star}=0\right)=-\frac{2 \hat{p_{1}^{\star}}}{\left(u_{\alpha}^{\star}-c^{\star}\right) p_{\alpha}^{\star}} \exp \left[i a^{\star}\left(x^{\star}-c^{\star} t^{\star}\right)\right]
$$

Notice in particular that in the case of plane waves $\left(\lambda^{\star}=0\right)$,

$$
\begin{aligned}
c^{\star} & =U_{\infty}^{*}\left[1 \pm \frac{1}{M_{\infty}}\right] \\
v_{1}^{*} & =0,
\end{aligned}
$$

(whilst (2.15) is unaltered).

The slip velocity is reduced to zero, in the usual way, by the inclusion of a thin boundary layer. The boundary layer approximation reduces the governing equations to (Stewartson 1964)

$$
\rho^{\star}\left[\frac{\partial u^{\star}}{\partial t^{\star}}+u^{\star} \frac{\partial u^{\star}}{\partial x^{\star}}+v^{\star} \frac{\partial u^{\star}}{\partial y^{\star}}\right]=-\frac{\partial p^{\star}}{\partial x^{\star}}+\frac{\partial}{\partial y^{\star}}\left(\mu^{\star} \frac{\partial u^{\star}}{\partial y^{\star}}\right)
$$




$$
\begin{aligned}
& \qquad \frac{\partial \rho^{\star}}{\partial t^{\star}}+\frac{\partial}{\partial x^{\star}}\left(p^{\star} u^{\star}\right)+\frac{\partial}{\partial y^{\star}}\left(\rho^{\star} v^{\star}\right)-0, \\
& \left.\rho^{\star} c_{p}\left[\frac{\partial T^{\star}}{\partial t^{\star}}+u^{\star} \frac{\partial T^{\star}}{\partial x^{\star}}+v^{\star} \frac{\partial T^{\star}}{\partial y^{\star}}\right]-\frac{\partial p^{\star}}{\partial t^{\star}}-u^{\star} \frac{\partial p^{\star}}{\partial x}-\frac{\partial}{\partial y^{\star}} \mid \mu^{\star} \frac{c p}{\sigma} \frac{\partial T \star}{\partial y^{\star}}\right]+\mu^{\star}\left[\frac{\partial u^{\star}}{\partial y^{\star}}\right]^{2}, \\
& \text { (together with (2.1)). } \\
& \qquad \frac{\partial p^{\star}}{\partial y^{\star}}-0, \\
& \text { These equations can be simplified by the use of a generalisation } \\
& \text { of the Howarth-Dorodnitsyn transformation, suggested by Stewartson (1951) } \\
& \text { and Moore (1951). We write }
\end{aligned}
$$

$$
\rho^{\star}=\rho_{\infty}^{\star} \frac{\partial \bar{Y}}{\partial y^{\star}}, \quad u^{\star}-\frac{\partial \psi^{*}}{\partial \bar{Y}}
$$

where $\bar{Y}$ now replaces $y^{\star}$ as the independent transverse variable, and $i^{*}$ is essentially a streamfunction. Then

$$
v^{\star}=-\frac{\rho_{\alpha}^{\star}}{\rho^{\star}}\left[\left[\frac{\partial \psi^{\star}}{\partial x^{\star}}\right]_{\bar{Y}, t}+u^{\star}\left[\frac{\partial \bar{Y}}{\partial x^{\star}}\right]_{y^{\star}, t^{\star}}+\left[\frac{\partial \bar{Y}}{\partial t^{\star}}\right]_{x^{\star}, y^{\star}}\right]
$$

and the equations of motion and energy reduce to

$$
\begin{aligned}
& \frac{\partial^{2} \psi^{\star}}{\partial \bar{Y} \partial t^{\star}}+\frac{\partial \psi^{*}}{\partial \bar{Y}} \frac{\partial^{2} \psi^{\star}}{\partial x^{\star} \partial \bar{Y}}-\frac{\partial^{2} \psi^{\star}}{\partial \bar{Y}^{2}} \frac{\partial \psi^{*}}{\partial x^{\star}}
\end{aligned}
$$

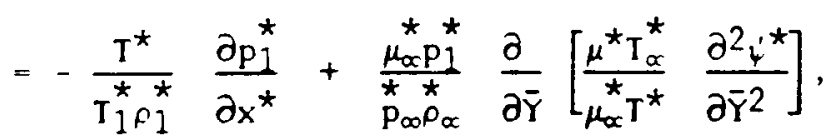




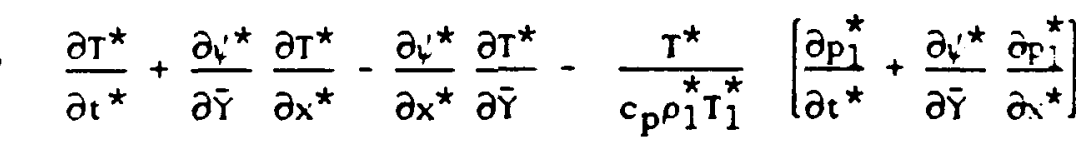

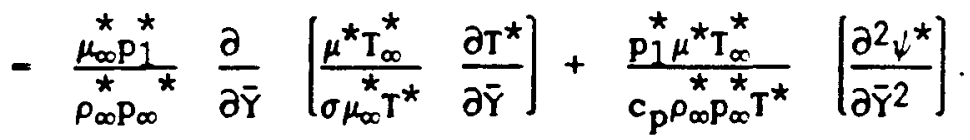

On account of $(2.21)$, we have written

$$
\mathrm{p}^{\star}\left(\mathrm{x}^{\star}, \overline{\mathrm{Y}}, \mathrm{t}^{\star}\right)=\mathrm{p}_{1}^{\star}\left(\mathrm{x}^{\star}, \mathrm{t}^{*}\right)
$$

The usage of the streamfunction ensures that the cont inuity condition $(2,19)$ is satisfied.

We now seek a solution to (2.24) - (2.25), subject to the boundary conditions

$$
\begin{gathered}
\frac{\partial T^{\star}}{\partial \bar{Y}}(\bar{Y}-0)=0, \\
T^{\star}(\bar{Y} \rightarrow \infty) \rightarrow 1+0(\varepsilon), \\
\psi^{\star}(\bar{Y}-0)=\psi^{*}(\bar{Y}-0)=0, \\
\psi_{\bar{Y}}^{\star} \rightarrow U_{\infty}^{\star}-\frac{2 \hat{p}_{1}^{\star} \varepsilon}{\left(U_{\infty}^{\star}-c^{\star}\right) \rho_{\infty}^{*}} \exp \left[j \alpha^{\star}\left(x^{\star}-c^{\star} t^{\star}\right)\right] \text { as } \bar{Y} \rightarrow \infty .
\end{gathered}
$$

At this stage we must specify the particular viscosity/temperature model, and here we choose the simplest example, namely the linear law of Chapman (See Stewartson 1964),

$$
\mu^{\star}=\frac{\mu_{o c}^{*} T^{\star}}{T_{\infty}^{\star}}
$$

We now seek a perturbation solution (in powers of $\varepsilon$ ); we write 


$$
\begin{aligned}
& \psi^{\star}-\psi_{0}^{\star}+\epsilon \bar{\psi}^{\star}+\ldots \\
& T^{\star}=T_{0}^{\star}+\epsilon \overline{\mathrm{T}}^{\star}+\ldots \\
& \rho^{\star}-\rho_{0}^{\star}+\epsilon \bar{\rho}^{\star}+\ldots \\
& \bar{Y}=Y_{0}+\epsilon \bar{Y}+\ldots \\
& \mathrm{p}_{1}=\rho_{\infty}^{\star} \mathrm{R}_{\infty}^{\star}+\epsilon \tilde{\mathrm{P}}^{\star}+\ldots
\end{aligned}
$$

Substituting $(2.32)^{*}$ into $(2.24)-(2.25)$ yields, to $O\left(\epsilon^{0}\right)$

$$
\frac{\partial \psi_{0}^{\star}}{\partial Y_{0}} \frac{\partial^{2} \psi_{0}^{\star}}{\partial x^{\star} \partial Y_{0}}-\frac{\partial \psi_{0}^{\star}}{\partial x^{*}} \frac{\partial^{2} \psi_{0}^{*}}{\partial Y_{0}^{2}}-\frac{\mu_{\infty}^{*}}{\rho_{\infty}^{\star}} \frac{\partial^{3} \psi_{0}^{*}}{\partial Y_{0}^{3}}
$$

$\frac{\partial \psi_{0}^{*}}{\partial Y_{0}} \frac{\partial \mathrm{T}_{0}^{\star}}{\partial \mathrm{x}^{*}}-\frac{\partial \psi_{0}^{\star}}{\partial \mathrm{x}^{*}} \frac{\partial \mathrm{T}_{0}^{\star}}{\partial \mathrm{Y}_{0}}-\frac{\mu_{\infty}^{\star}}{\sigma \rho_{\infty}^{\star}} \frac{\partial^{2} \mathrm{~T}_{0}^{\star}}{\partial \mathrm{Y}_{0}^{2}}+\frac{\mu_{\infty}^{\star}}{\mathrm{c}_{\mathrm{p}^{p_{\infty}^{*}}}^{\star}}\left[\frac{\partial^{2} \psi_{0}^{\star}}{\partial \mathrm{Y}_{0}^{2}}\right]^{2}$

The solution of $\psi_{0}^{\star}$ (and $\mathrm{T}_{0}^{\star}$ ) is now rout ine, and merely corresponds to the similarity form of Blasius. Writing

$$
\begin{aligned}
& \psi_{0}^{\star}=\left[\frac{2 \mu_{\infty}^{\star} U_{\infty}^{\star} x^{\star}}{\rho_{\infty}^{\star}}\right]^{\frac{1}{2}} F_{0}(\eta), \\
& T_{0}^{\star}=T_{\infty}^{*} G_{0}(\eta),
\end{aligned}
$$

where $\eta=Y_{0}\left[\frac{\rho_{\alpha}^{\star} U_{\infty}^{\star}}{2 \mu_{\infty}^{\star} x^{\star}}\right]^{\frac{1}{2}}$,

then

$$
\begin{gathered}
F_{0}^{\prime \prime}+F_{0} F_{0}^{\prime \prime}-0, \\
\frac{1}{\sigma} C_{0}^{\prime \prime}+F_{0} C_{0}^{\prime}+(\gamma-1) M_{\infty}{ }^{2} F_{0}{ }^{n}=0 .
\end{gathered}
$$


The boundary conditions to be applied are

$$
\begin{aligned}
& F_{0}(0)=F_{0}^{\prime}(0)=0, \\
& F_{0}(\infty)=1 \\
& G_{0}^{\prime}(0)=0 \\
& C_{0}(\infty)=1 .
\end{aligned}
$$

The $O(\varepsilon)$ equations are rather more complicated, although their derivation is routine. The momentum and energy equations at this order are, respectively

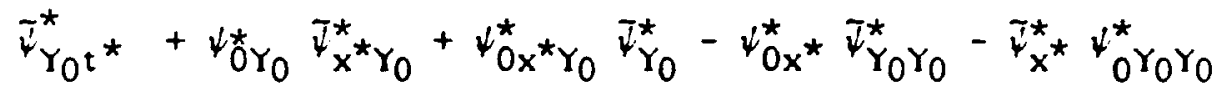

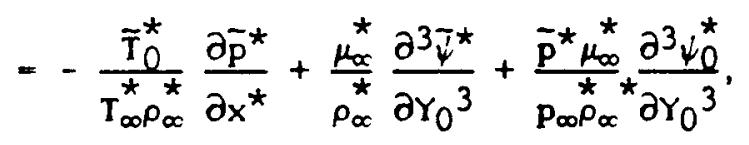

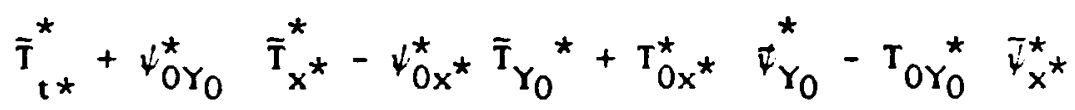

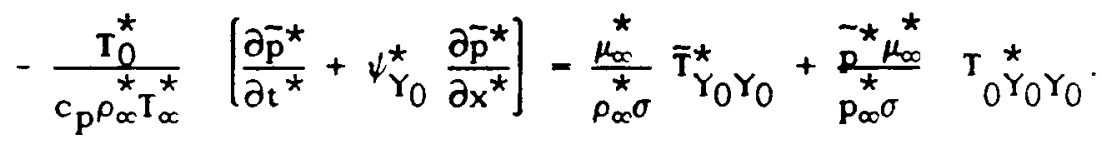


Our primary concern here will be with the momentum equation (2.41) (although a similar treatment may be carried out on (2.42)).

We already have $(2.35)-(2.37)$, and now we write

$i^{\star}=-\frac{2 \hat{p}_{1}^{\star}}{\left(u_{\infty}^{\star}-c^{\star}\right) \rho_{\alpha}^{\star}}\left[\frac{2 \mu_{\infty}^{\star} x^{\star}}{\rho_{\infty}^{\star} u_{\infty}^{\star}}\right)^{\frac{1}{2}} F_{1}(\eta, \xi) e^{-i \alpha^{\star} c^{\star} t^{\star}}$,

where $\xi-x^{\star} \frac{1}{2}$.

These transformations, when applied to (2.41) yield the following equation for $F_{1}(\eta, \xi)$,

$F_{1 \eta \eta \eta}+F_{0} F_{1 \eta \eta}+F_{1} F_{0 \eta \eta}+\frac{2 i \alpha^{\star} c^{\star}}{u_{\infty}^{\star}} \xi^{2} F_{1 \eta}-\xi F_{0 \eta} F_{1 \eta \xi}+\xi F_{1 \xi} F_{0 \eta \eta}$

$-2 C_{0}(\eta) \xi^{2} i \alpha\left[\frac{c^{\star}}{U_{c}^{\star}}-1\right] e^{i \xi^{2} \alpha^{\star}}-\gamma M_{\infty}^{2}\left[\frac{c^{\star}}{U_{\infty}^{\star}}-1\right] e^{i \xi^{2} \alpha^{\star}} F_{0 \eta \eta \eta}$

$a^{\star}$ may be completely eliminated from the problem, by introducing the new scaled variable $\hat{\xi}$

$$
\hat{\xi}=\left[\frac{c^{\star} \alpha^{\star}}{u_{\alpha}^{\star}}\right]^{\frac{1}{2}} \xi
$$

It is also convenient to introduce the non-dimensional wavespeed,

$$
c=c^{*} / U_{\infty}^{*}
$$

and so (2.41) becomes

$$
\begin{aligned}
& F_{1 \eta \eta \eta}+F_{0} F_{1 \eta \eta}+F_{1} F_{0 \eta \eta}+2 i \hat{\xi}^{2} F_{1 \eta}-\hat{\xi} F_{0 \eta} F_{1 \eta \hat{\xi}}+\hat{\xi} F_{1 \hat{\xi}} F_{0 \eta \eta} \\
& =2 i C_{0}(\eta) \hat{\xi}^{2}\left(1-\frac{1}{c}\right) e^{i \hat{\xi}^{2} / c}-\gamma M_{\infty}^{2}(c-1) e^{i \hat{\xi} 2 / c} F_{0 \eta \eta \eta},
\end{aligned}
$$

subject to

$$
\begin{aligned}
& F_{1}(r-0)=F_{1 \eta}(\eta-0)=0 \\
& F_{1 r_{1}} \rightarrow e^{i \hat{\xi}^{2} / c} \text { as } \eta \rightarrow \alpha .
\end{aligned}
$$


This equation may be viewed as the compressible counterpart of the work of Patel (1975), and also of Ackerberg and Phillips (1972) (and others cited previously) which was specifically concerned with temporal oscillations.

Equations (2.38)-(2.39) were solved numerically, using second-order finite-difference scheme, in which the momentum and energy equations were split into three and two first order ordinary differential equations respectively, and solved iteratively using Newton's method. $F_{0}(\eta)$ and $\mathrm{G}_{0}(\eta)$ were then input into (2.48); this (1Inear) equation was solved using second order finite-differences in $\eta$, Crank-Nicolson marching in $\hat{\xi}$, again by splitting the equation into a system of first order equations (in $\eta$ ).

Results for $e^{-i \hat{\xi}^{2} / c_{F}} F_{1 \eta \eta}(\eta-0) / \hat{\xi}$ are shown in Figs $1-4$. In a11 cases we set $\gamma=1.4, \sigma=0.72, \lambda=0$ (plane waves) and the results are believed to be correct to within the graphical tolerance of the figures. Figures $1 \mathrm{a}, \mathrm{b}$ show results for $M_{\infty}=\sqrt{2}, \quad c=1-\frac{1}{M_{\infty}}$, Figs. 2 a,b for $M_{\infty}=/ 2, \quad c=1+1 / M_{\infty}$, Figs $3 a, b$ for $M_{\infty}-5, c=1-1 / M_{\infty}$, Fig. $4 a, b$ for $M_{x}=5, c=1+1 / M_{x}$.

It is readily apparent from these results that as $\xi \rightarrow \infty$ (i.e. far downstream), $\quad e^{-i \hat{\xi} 2 / c} F_{1 \eta \eta}(\eta-0) / \hat{\xi}$ asymptotes to a constant, and it is a simple matter to confirm this analytically.

From (2.48), we have as $\xi \rightarrow \infty$

$$
2 i \hat{\xi}^{2} F_{1 \eta}-\hat{\xi} F_{0} F_{1 \eta \hat{\xi}}+\hat{\xi} F_{1} \hat{\xi}_{0 \eta \eta}=2 i C_{0}(\eta) \hat{\xi}\left(1-\frac{1}{c}\right) e^{i \hat{\xi}^{2} / c} \text {. }
$$

The inclusion of the second and third terms here is most easily just ified by transforming to the (non-dimensional) streamwise ( $x$ ) coordinate, where

$$
x=\hat{\xi}^{2},
$$


whence

$$
i F_{1 \eta}-F_{0 \eta} F_{1 \eta x}+F_{0 \eta \eta} F_{1 x}-i C_{0}(\eta)\left(1-\frac{1}{c}\right) e^{i x / c}
$$

Writing

$$
F_{1}(\eta, x)-\hat{F}_{1}(\eta) e^{i x / c}
$$

then

$$
\hat{F}_{1}(\eta)=\left(c-F_{0 \eta}\right) \int_{0}^{\eta} \frac{G_{0}(\eta)(c-1)}{\left[c-F_{0 \eta}\right]^{2}} d \eta
$$

This solution generally violates the no-slip condition on $r 0$, since

$$
\hat{\mathbf{F}}_{1 \eta}(\eta-0)-\mathrm{G}_{0}(0)\left(1-\frac{1}{c}\right) \text {. }
$$

However this is easily rectified by the Inclusion of a Stokes layer, where

$$
Y=\eta \xi-\eta \times^{\frac{1}{2}}-0(1)
$$

and

$$
F_{1 Y Y Y}+2 i F_{1 Y}=2 i C_{0}(0)\left(1-\frac{1}{c}\right) e^{i x / c} .
$$

Writing

$$
F_{1}(Y, X)-\hat{f}_{1}(Y) e^{i x / c}
$$

then

$$
\hat{f}_{1 Y}(Y)=G_{0}(0)\left(1-\frac{1}{c}\right)\left[1-e^{-(1-i) Y}\right]
$$

which does sat isfy the no-slip condition on Y=0. Equation (2.59) then gives

$$
F_{1 \eta \eta}(\eta-0)-\hat{\xi} e^{i \xi^{2} / c} C_{0}(0)\left(1-\frac{1}{c}\right)(1-i)
$$

This is shown as an asymptote on Figs. 1-4, and the result is seen to be confirmed. Equation (2.54) does adrit the possibility of a 
singularity, wherever

$$
F_{0 \eta}-1 / c
$$

and a critical layer is necessary if this occurs, as described in the Appendix.

Unfortunately the above expansion is not uniformly valid as $\xi \rightarrow \infty$. This is most easily seen by comparing the magnitude of the transverse pressure gradient $\left(\partial p^{\star} / \partial y^{*}\right)$ with the transverse inertia terms (in particular the $\rho^{\star} u^{\star} v_{x}^{\star}$ term) namely.

$$
\begin{aligned}
\frac{\frac{\partial p^{*}}{\partial y^{*}}}{\rho^{\star} u^{\star} v^{*}}-0\left[\frac{\rho_{\infty}^{\star} U_{\infty}^{\star 2}\left[\frac{\rho_{\infty}^{\star} U_{\infty}^{\star}}{\mu_{\infty}^{\star} x^{\star}}\right]^{\frac{1}{2}}}{\rho_{\infty}^{\star} \alpha^{\star} 2 U_{\infty}^{\star}\left[\frac{\mu_{\infty}^{\star} U_{\infty}^{\star} x^{\star}}{\rho_{\infty}^{\star}}\right]^{\frac{1}{2}}}\right] \\
=0\left[\frac{\rho_{\infty}^{\star} U_{\infty}^{\star}}{\mu_{\infty}^{\star} x^{\star} \alpha^{\star 2}}\right] .
\end{aligned}
$$

Hence there will be a breakdown to the above solution when

$$
x^{\star}=0\left[\frac{\rho_{\infty}^{\star} U_{\infty}^{\star}}{\mu_{\infty}^{\star} \alpha^{\star 2}}\right],
$$

when the transverse pressure gradient can no longer be neglected. In the following section we go on to consider the repercusions of this.

One final point is that eigensolutions analogous to those described by Lam and Rott (1960) are certainly expected to occur far downstream (although their magnitude was such that they were undetectable in our numerical restits); however, as noted in the previous section, unlike the subsonic case. in the supersonic planar case currently under consideration, these eigensolutions are of little consequence. 


\section{The far downstream region}

As noted at the end of the last section, unsteady transverse pressure fluctuations will become significant in the physics of the boundary layer when

$$
x^{\star}=0\left[\frac{\rho_{\infty}^{\star} U_{\infty}^{\star}}{\mu_{\infty}^{\star} \alpha^{\star 2}}\right] .
$$

This implies (and is consistent with)

$$
y^{*}-0\left(\alpha^{\star-1}\right)
$$

implying the transverse scale (i.e. the boundary layer thickness) is comparable with the wavelength of the imposed wave. At the same time, the streamwise lengthscale of the wave $\left(\alpha^{*-1}\right)$ is much shorter than the developmental length of the boundary layer (1.e. (3.1)), and so the parallel flow approximation is a rational procedure, and is adopted here.

$$
\begin{aligned}
& \text { We choose to make the following non-dimensionalisations } \\
& y=y^{\star} / \delta^{\star} \\
& \alpha=\alpha^{\star} \delta^{\star} \\
& (u, v)=\left(u^{\star}, v^{\star}\right) / U_{\infty}^{\star}, \\
& \rho=\rho^{\star} / \rho_{\infty}^{\star} \\
& P=p^{\star} /\left(\rho_{\infty}^{\star} R^{\star} T_{\infty}^{\star}\right) \\
& T=T^{\star} / T_{\infty}^{\star} \\
& c=c^{\star} / U_{\infty}^{*} \\
& t=\frac{U_{\alpha}^{\star} t^{\star}}{\delta^{\star}} .
\end{aligned}
$$

Here $\delta^{*}$ represents the boundary layer thickness, (and is the key lengthscale used in the non-dimensionalisation process), i.e. 


$$
\delta^{*}-\left[\frac{2 \mu_{\infty}^{*} x^{*}}{\rho_{\infty}^{*} u_{\infty}^{*}}\right]^{\frac{1}{2}}
$$

We then expect the solution to develop as follows

$$
\begin{aligned}
& u=\bar{U}(y)+\epsilon f(y) E+\ldots \\
& v-\varepsilon \alpha \varphi(y) E+\ldots \\
& p=1+\varepsilon \pi(y) E+\ldots \\
& T=\bar{T}(y)+\varepsilon \theta(y) E+\ldots \\
& \rho-\bar{\rho}(y)+\varepsilon R(y) E+\ldots
\end{aligned}
$$

with $E=e^{i \alpha(x-c t)}$,

and where $\overline{\mathbf{U}}(y), \bar{\rho}(y)$ and $\overline{\mathrm{T}}(y)$ denote the mean flow speed, density density and temperature respectively.

The governing equations are then found to be (Lees and Lin 1946)

$$
\begin{aligned}
& \rho\left[i(\bar{U}-c) f+U^{\prime} \varphi\right]=\frac{-i \pi}{M_{\infty} z} \\
& \bar{\rho} i \alpha^{2}(\bar{U}-c) \varphi=\frac{-\pi^{\prime}}{\gamma M_{\infty}{ }^{2}} \\
& i(\bar{U}-c) R+\bar{\rho}\left(\varphi^{\prime}+i f\right)+\bar{\rho}^{\prime} \varphi=0, \\
& \pi-\frac{R}{\bar{\rho}}+\frac{\theta}{\bar{T}},
\end{aligned}
$$

$$
\left[\mathbf{i}(\overline{\mathbf{U}}-\mathrm{c}) \theta+\overline{\mathbf{T}}^{\prime} \varphi\right]-\mathbf{i}\left[\frac{\gamma-1}{\gamma}\right] \pi(\overline{\mathbf{U}}-\mathrm{c}) \overline{\mathbf{T}}
$$

After some simple manipulation (following Lees and Lin 1946), we arrive at the following equation for $\varphi$,

$$
\frac{\mathrm{d}}{\mathrm{dy}}\left\{\frac{(\overline{\mathrm{U}}-c) \varphi^{\prime}-\overline{\mathrm{U}}^{\prime} \varphi}{\overline{\mathrm{T}}-\mathrm{M}_{\infty}^{2}(\overline{\mathrm{U}}-c)^{2}}\right\}-\frac{\alpha^{2}(\overline{\mathrm{U}}-c) \varphi}{\overline{\mathrm{T}}}=0
$$

(1t is worth noting at this stage that the analogous governing equation of Mack 1984, 1987 appears inconsistent with this equation, and should be, 
In Mack's notation

$\left.D\left\{\frac{(\bar{\alpha} \overline{\mathrm{U}}-\omega) \hat{\mathrm{DV}}-\bar{\alpha} \mathrm{DU} \overline{\mathrm{V}}}{\left(1-\overline{\mathrm{M}}^{2}\right) \overline{\mathrm{T}}}\right\}-\frac{\bar{\alpha}^{2}(\bar{\alpha} \overline{\mathrm{U}}-\omega) \hat{\mathrm{V}}}{\overline{\mathrm{T}}}\right]$.

Finally, we follow the same approach as used previously, by using the transformation

$$
\frac{\partial \bar{Y}}{\partial y}-\bar{\rho}(y)
$$

(analogous to $(2.21)$ ) and so (3.11) becomes

$$
\frac{d}{d \bar{Y}}\left\{\frac{[\bar{U}(\bar{Y})-c] \varphi_{\bar{Y}}-\bar{U}^{\prime}(\bar{Y}) \varphi}{\bar{T}\left[\bar{T}-M_{\infty}{ }^{2}(\bar{U}(\bar{Y})-c)^{2}\right]}\right\}-\alpha^{2}(\bar{U}-c)_{\varphi}=0,
$$

where $U(\bar{Y})-F_{0}^{\prime}(\eta)$,

$$
\begin{gathered}
\overline{\mathbf{Y}}=\eta, \\
\overline{\mathrm{T}}(\overline{\mathrm{Y}})=\mathrm{G}_{0}(\eta),
\end{gathered}
$$

with $F_{0}(\eta)$ defined by (2.37), and $G_{0}(\eta)$ by $(2.38)$.

The boundary condition on $\bar{Y}=0$ is

$$
\varphi(\bar{Y}=0)=0
$$

corresponding to the impermeability condition. As $\bar{Y} \rightarrow \infty$, we require the match with the progressive wave solution. From the previous section, this condition can be described by the superposition of an incoming and outgoing wave, if

$$
\varphi-e^{i \alpha \lambda \bar{Y}}+\beta e^{-i \alpha \lambda \bar{Y}} \text { as } Y \rightarrow \infty
$$

$\beta$ is a reflection coefficient, which is to be determined as part of the solution. The wavespeed $c$ is given by 


$$
c-1 \pm \frac{1}{\mu_{\infty}}\left(1+\lambda^{2}\right) \frac{1}{2}
$$

on account of (2.13).

The problem then may be thought of as that to determine $\beta$, given $\lambda$ (generally complex), $M_{\infty}$, and $\alpha$. This was achieved by writing

$$
\varphi=\hat{\varphi}(\bar{Y})+e^{i \alpha \lambda \bar{Y}}
$$

where,

$$
\begin{aligned}
& \frac{d}{d \bar{Y}}\left\{\frac{[\bar{U}(\bar{Y})-c] \hat{\varphi}^{\prime}(\bar{Y})-\bar{U}^{\prime}(\bar{Y}) \hat{\varphi}}{T(Y)\left[T(Y)-M_{\infty}^{2}(U(Y)-c)^{2}\right]}\right\}-\alpha^{2}(\bar{U}(\bar{Y})-c) \hat{\varphi}(\bar{Y}) \\
& =e^{i \alpha \lambda \bar{Y}}\left\{\frac{\alpha^{2} \lambda^{2}(\bar{U}-c)+\bar{U}^{\prime \prime}(\bar{Y})}{\overline{\mathbf{T}}\left[\overline{\mathrm{T}}-M_{\infty}^{2}(\overline{\mathrm{U}}-c)^{2}\right]}+\frac{\overline{\mathrm{T}}^{\prime}(\overline{\mathrm{Y}})}{\overline{\mathrm{T}}^{2}}\left[\frac{i \alpha \lambda(\overline{\mathrm{U}}-\mathrm{c})-\overline{\mathrm{U}}^{\prime}(\overline{\mathrm{Y}})}{\left[\overline{\mathrm{T}}-\mathrm{M}_{\infty}^{2}(\overline{\mathrm{U}}-c)^{2}\right]}\right.\right. \\
& \left.\left.+\frac{\left[i \alpha \lambda(\bar{U}-c)-\bar{U}^{\prime}(\bar{Y})\right]\left[\overline{\mathbf{T}}^{\prime}-2 M_{c}^{2}(\bar{U}-c) \bar{U}^{\prime}(\bar{Y})\right]}{\overline{\mathbf{T}}\left[\overline{\mathrm{T}}-M_{\infty}^{2}(U-c)^{2}\right]^{2}}\right]\right\} \text {. }
\end{aligned}
$$

This equation was split into two first order equations, one for 
$\hat{\varphi}^{\prime}(\bar{Y})$, the other for $\hat{F}^{\prime}(\bar{Y})$, where

$$
\hat{F}(\bar{Y})=\frac{[\bar{U}(\bar{Y})-c] \hat{\varphi}^{\prime}(\bar{Y})-\bar{U}^{\prime}(\bar{Y}) \hat{\varphi}}{\bar{T}\left[\bar{Y}-M_{\infty}^{2}(\bar{U}(\bar{Y})-c)^{2}\right]}
$$

A simple fourth-order Runge-Kutta method was then used. To obtain the appropriate behaviour at infinity, the radiation condition

$$
\hat{\varphi}_{\bar{Y}}+1 \alpha \hat{\varphi}_{\varphi_{2}}=0
$$

was applied at some sultably large value of $\tilde{Y}$.

This solution will in general fail to satisfy the no-slip condition; however this is easily remedied by the presence of a stokes layer of thickness $\bar{Y}=O(1) \quad($ see $(2.56))$.

Figures 5-10 show the variation of the reflection coefficient $\beta$ (see (3.12)) with $\alpha$. Results for $M_{\infty}-J 2, \lambda-\frac{1}{2}+\frac{1}{2} i$, negat ive root of (3.17) are shown in Figs. 5a,b; for $M_{\infty}=J 2, \lambda=\frac{1}{4}+\frac{1}{4} i$ negative root of (3.17) in Figs. 6a,b; for $M_{\infty}=J 2 \lambda-\frac{1}{2}+\frac{1}{2} i$, positive root of (3.17) in Figs. 7a,b; for $M_{\infty}=J 2, \lambda=\frac{1}{4}+\frac{1}{4} i$, positive root of of (3.17) in Figs. 8a,b; for $M_{\infty}=5, \lambda-\frac{1}{2}+1$, negative root of (3.17) in Fig. 9a,b; for $M_{\infty}-5, \lambda=\frac{1}{4}+\frac{1}{4} i$, negative root of (3.17) in

Figs. 10a,b. All these results were obtained with $\gamma=1.4, \sigma=0.72$ :

The primary result which is clearly visible from these computations is that $\beta \rightarrow-1$ as $\alpha \rightarrow 0$. This indicates a correct match with the results of the previous section in the upstream limit $(\alpha \rightarrow 0$, here, may be interpreted as the upstream limit), in particular with (2.15). This match will be studied in full in the following section.

$$
\text { Lastly, it is also clear that as } \alpha \rightarrow \infty \text { (downstream } 1 \mathrm{fmit} \text { ), } \beta \rightarrow 0 \text {. }
$$

In the following section we summarise the conclusions of this work. 


\section{The matching of the fore and downstream regions}

Let us first consider the limit of (3.11) as $\alpha \rightarrow 0$ (which as pointed out at the end of the previous section is equivalent to the upstream limit of the downstream zone). Two lengthscales for $y$ emerge, the first, where

$$
\hat{y}-\alpha y=0(1)
$$

involves a solution to (3.11) of an incoming and outgoing wave, namely

$$
\varphi=e^{i \hat{\lambda y}}+\beta e^{-i \hat{\lambda y}}+O\left(\alpha^{0}\right) \text {. }
$$

We may impose the impermeability constraint on this system, which requires $\beta=-1$ and so to leading order

$$
\varphi=e^{i \lambda \hat{y}}-e^{-i \hat{\lambda y}}
$$

this condition matching correctly with the outer fore-region solution $(2.14)$.

The second important lengthscale is $y-0(1)$ itself, where the variation of $\overline{\mathbf{T}}$ and $\overline{\mathbf{U}}$ must be taken into consideration. To leading order we have

$$
\frac{d}{d y}\left\{\frac{(\bar{U}-c) \varphi^{\prime}-\bar{U}^{\prime} \varphi}{\bar{T}-M_{\infty}^{2}(\bar{U}-c)^{2}}\right\}=0 .
$$

The solution to this is

$$
\varphi=C(\overline{\mathrm{U}}-\mathrm{c}) \int_{0}^{\mathrm{y}} \frac{\overline{\mathbf{I}}-\mathrm{M}_{\infty}(\overline{\mathrm{U}}-\mathrm{c})^{2}}{(\overline{\mathrm{U}}-\mathrm{c})^{2}} d y,
$$

where the impermeability condition has been imposed, and $C$ is a 
constant which is determined by atching with (4.3), which yields

$$
c=-\frac{2 \alpha !(1-c)}{\lambda}
$$

This completes the details of the $\alpha \rightarrow 0$ solution; however for the purposes of matching with the fore-region, the simplest illustration is by means of the perturbation streamwise velocity $f(y)$. By (3.6) we see we may wite

$$
f(\because)=-\frac{-x \bar{T}}{M_{\infty}^{2}(\bar{U}-c)}+\frac{i_{\bar{U}}^{\prime} \varphi}{(\bar{U}-c)} .
$$

The pressure term $x$ is (by (3.7)) seen to be independent of $y$, and given by

$$
\pi=-i \mathrm{C} \mathrm{M}_{\infty}{ }^{2}
$$

and so

$$
\begin{array}{r}
f(y)=\frac{i c \overline{\mathrm{T}}}{\overline{\mathrm{U}}-\mathrm{c}}+\mathrm{i} \overline{\mathbf{L}}^{\prime} \mathrm{c} \int_{0}^{\mathrm{y}} \frac{\overline{\mathbf{T}}}{(\overline{\mathrm{U}}-\mathrm{c})^{2}} \mathrm{dy} \\
-i \overline{\mathrm{U}}^{\prime} \mathrm{am}_{\infty}{ }^{2} \mathrm{y}
\end{array}
$$

(if $\bar{U}-c$, then a critical layer of the type described in the Appendix will be present).

We now match the downstream limit of the fore-region with (4.9)

The $O(\varepsilon)$ perturbation to the $x^{\star}$ component of velocity, as $x^{\star} \rightarrow \infty$ may be written

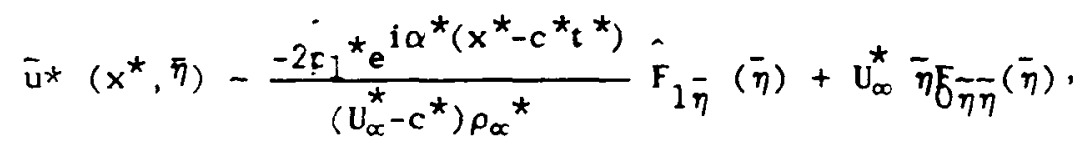


where $\bar{\eta}$ is defined by

$$
\bar{\eta}-\overline{\mathrm{Y}}\left[\frac{\rho_{\infty}^{\star} \mathrm{U}_{\alpha}^{\star}}{2 \mu_{\infty}^{*} x^{\star}}\right]^{\frac{1}{2}}
$$

whilst

$$
\bar{\eta}(\bar{\eta})-\int_{0}^{\bar{\eta}} \frac{\rho^{\star}(\bar{\eta})-\rho_{0}^{\star}(\bar{\eta})}{\left.\rho_{C}^{\star} \bar{\eta}\right)} \mathrm{d} \bar{\eta} .
$$

and $\hat{F}_{1}(\bar{\eta})$ is given by $(2.54)$ (with $\eta$ replaced by $\bar{\eta}$ ). Notice that

$$
\bar{\eta}=\int_{0}^{y} \frac{\rho^{\star}}{\rho_{\infty}} d y .
$$

Equation (4.12) may be written

$$
\tilde{\eta}(y)=\int_{0}^{y}\left[\frac{\tilde{p}^{*}}{\rho_{\infty}{ }^{*} R^{*} T_{\infty}{ }^{*} C_{0}(y)}-\frac{\theta_{I}^{*}(y)+\theta_{2}{ }^{*}(y)}{T_{\infty}{ }^{*} C_{0}^{2}(y)}\right] d y .
$$

Here $\theta_{1}^{*}(y)=\tilde{\eta} T_{\infty}^{\star} G_{0 y}(y) G_{0}(y)$,

whilst $\theta_{2}^{*}(y)$ is determined from the far downstream limit of (2.42)

which yields

$$
\theta_{2}^{\star}(y)=\frac{G_{0}(y) \tilde{p}^{\star}}{C_{p} \rho_{\infty}^{\star}}+\frac{T_{=}^{\star} C_{0 y} \hat{F}_{1}(y) E^{\star} C_{1}}{U_{0}^{*}-c^{\star}}
$$

where we have written

$$
E^{\star}=e^{i a^{\star}\left(x^{\star}-c^{\star} t^{*}\right)}
$$




$$
\begin{aligned}
& U_{0}^{\star}(y)-U_{\infty}^{*} U_{0}(y)-U_{\infty}^{\star} F_{0}^{j}(\bar{\eta}), \\
& c_{1}-\frac{2 \hat{p}_{1}^{\star}}{\left(U_{\infty}^{\star}-c^{\star}\right) \rho_{\infty}^{\star}} .
\end{aligned}
$$

Consequently we see that

$\tilde{\eta}(y)=\int_{0}^{y}\left[\frac{\tilde{p}^{\star}}{\rho_{\infty}^{\star} R^{\star} T_{\infty}^{\star} C_{0}(y)}-\frac{\tilde{\eta}(y) C_{0 y}(y)}{C_{0}}-\frac{\tilde{p}^{\star}}{T_{\infty}^{\star} c_{p_{\infty}}^{\star} \rho_{\infty} C_{0}(y)}-\frac{C_{0 y} \hat{F}_{1}(y) E^{\star} C_{1}}{C_{0}\left(U_{0}^{*}-c^{\star}\right)}\right] d y$.

The solution of this equation is

$\bar{\eta}(y)-\frac{1}{G_{0}(y)} \int_{0}^{y}\left\{\frac{\tilde{p}^{\star}}{\rho_{\infty}^{\star} R^{\star} T_{\infty}^{\star}}-\frac{\tilde{p}^{\star}}{T_{\infty}^{\star} c_{p} \rho_{\infty}^{\star}}-\frac{G_{0} \hat{F}_{1}(y) E^{\star} C_{1}}{U_{0}^{\star}-c^{\star}}\right\} d y$.

We now see that as $x^{*} \rightarrow \infty$

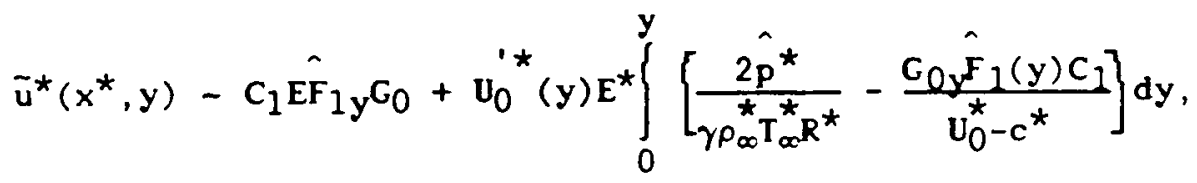

where we have used the condition

$$
\bar{p}^{\star}-2 \hat{p^{\star} E^{\star}}
$$

Equation (4.22) may now be written

$\tilde{u}^{\star}\left(x^{\star}, y\right)-\frac{C_{1} E^{\star} C_{0}(y)(c-1)}{c-U_{0}(y)}+\frac{2 U_{0}^{1}(y) E^{*} \hat{p y y}^{\star}}{\gamma \rho_{\infty}^{\star} T_{\infty}^{\star} R^{\star}}-C_{1} E^{\star} U_{0}^{1}(y)(c-1) \int_{0}^{y} \frac{C_{0}(y) d y}{\left(c-U_{0}\right)^{2}}$

$$
-\frac{c_{1} E^{\star} C_{0}(y)(c-1)}{c-U_{0}(y)}-c_{1} M_{\alpha}^{2} E^{\star} y U_{0}^{\prime}(y)(1-c)-C_{1} E^{\star} U_{0}^{\prime}(y)(c-1) \int_{0}^{y} \frac{C_{0}(y)}{\left(c-U_{0}\right)^{2}} d y .
$$




$$
\text { Setting } c_{1}-1 U_{\infty}^{*} C /(1-c) \text {. }
$$

and noting that

$$
\begin{aligned}
& C_{0}(y)-\bar{T}(y), \\
& U_{0}(y)=\bar{U}(y),
\end{aligned}
$$

we see that (4.25) matches correctly with (4.9). 


\section{Discussion and concluslons}

In this paper a description of the effect of a small amplitude progressive wave on a supersonic viscous flow over semi-infinite flat plate has been given. The original motivation in this work was to study the possibility of a supersonic analogy of the so-called receptivity problem; however it turns out that in this case there is no receptivity, at least in the absence of boundary curvature. However, it would certainly be possible that a higher order analysis incorporating boundary-layer growth terms (i.e. non-parallel effects) would give an element of boundary curvature.

Associated with the present study, the form of the compressible Stokes layer has been described, together with the ultimate breakdown of the (boundary layer) structure of the perturbation solution which far downstream becomes predominant ly inviscid. The analysis, together with our numerical results, formally indicate a proper match between the two regimes. 


\section{Appendix}

Here we consider the critical layer which wlll occur if

$$
F_{0 \eta}-c
$$

in (2.54). Since $0<F_{0 \eta}<1$, this situation is only possible if the negative root of (2.12) is chosen.

The treatment of the critical layer is fairly standard, and follows closely the structure found in, for example, linear stabllity theory (see for example Rosenhead 1963); as a consequence we shall keep details to a minimum.

Suppose that we have line, $\eta-\eta_{c}$, such that

$$
F_{0 \eta}\left(\eta-\eta_{c}\right)-c ;
$$

we then write

$$
F_{1}(\eta, x)=e^{i x / 2} \hat{F}_{1}(\eta)
$$

and then taking the inviscid equation (2.52), and performing a Frobenius expansion about $\eta=\eta_{c}$, gives, for $\eta<\eta_{c}$

$$
\begin{aligned}
\hat{F}_{1}(\eta)-\frac{G\left(\eta_{c}\right)(c-1)}{F_{0}^{\prime \prime}\left(\eta_{c} !\right.}+\frac{(c-1)}{F_{0}^{n}(\eta)}\left(\eta_{c}-\eta\right) \ln \left(\eta_{c}-\eta\right) & \left\{G_{0}^{\prime}\left(\eta_{c}\right)-\frac{F_{0}^{n \prime}\left(\eta_{c}\right) C_{0}\left(\eta_{c}\right)}{F_{0}^{n}\left(\eta_{c}\right)}\right\} \\
& +0\left(\eta_{c}-\eta\right) .
\end{aligned}
$$

For $\eta>\eta_{c}$ the same procedure yields

$$
\begin{array}{r}
\hat{F}_{1}(\eta)-\frac{G\left(\eta_{c}\right)(c-1)}{F_{0}^{n}\left(\eta_{c}\right)}+\frac{(c-1)}{F_{0}^{n}\left(\eta_{c}\right)}\left(\eta_{1}-\eta\right)\left[\ell n\left|\eta_{c}-\eta\right| \pm i x\right] \\
\left.G_{0}^{\prime}\left(\eta_{c}\right)-\frac{F_{0}^{\prime \prime \prime}\left(\eta_{c}\right) G_{0}\left(\eta_{c}\right)}{F_{0}^{n}\left(\eta_{c}\right)}\right\} \\
+0\left(\eta_{c}-\eta\right) .
\end{array}
$$

The ambiguity in sign here must be resolved by incorporating the effects of viscosity inside the critical layer. The similarity with linear 
incompressible stability theory is imediately apparent, the chief

difference being the inclusion of the $\dot{C}_{j}\left(\eta_{c}\right)$ term, i.e. the temperature gradient term, which is obviously absent in incompressible situations.

Following classical stability theory, we expect that inside the critical layer the solution develops as follows (assuming $x>>1$ )

$F_{1}(\eta, x)-e^{i x / c}\left\{\hat{F}_{10}(\zeta)+x^{-1 / 3} \hat{F}_{11}(\zeta)+\ldots\right\}$

where $r=\left(\eta_{c}-\eta\right) x^{1 / 3}$

- $0(1)$ inside the critical layer.

The governing equation for $\hat{F}_{1}(\zeta)$ is found to be (from (2.48))

$$
\begin{aligned}
& \hat{F}_{10}^{\prime \prime}-\frac{2 i}{c} \hat{F}_{0}^{n}\left(\eta_{c}\right) \hat{\zeta F_{10}^{\prime}}+\frac{2 i}{c} \hat{F}_{0}^{n}\left(\eta_{c}\right) \hat{F}_{10} \\
& =2 i G_{0}\left(\eta_{c}\right)\left(1-\frac{1}{c}\right)
\end{aligned}
$$

The solution to (A.8), which matches on to both (A.4) and (A.5) is simply

$$
\hat{F}_{10}-\frac{c_{0}\left(\eta_{c}\right)(c-1)}{F_{0}^{n}\left(\eta_{c}\right)} .
$$

The equation for $\hat{F}_{11}(\zeta)$ is found to be

$\hat{F}_{11}^{n}-\frac{2 i}{c} \hat{F}_{0}^{n}\left(\eta_{c}\right) \hat{\zeta F}_{11}^{\prime}+\frac{2 i}{c} \hat{F}_{0}^{n}\left(\eta_{c}\right) \hat{F}_{11}-2 i \dot{G}_{0}^{\prime}\left(\eta_{c}\right) \zeta\left(1-\frac{1}{c}\right)-\frac{2 i}{c} \hat{F}_{10} \zeta \hat{F}_{0}^{\prime \prime}\left(\eta_{c}\right)$.

The solution of this may be expressed in terms of the functions due to Holstein (1950) (alternatively in terms of Airy functions) and this demands that if a proper matching is to be achieved with (A.4) as $\zeta \rightarrow \infty$, and $($ A.5) as $\zeta \rightarrow-\infty$, then we must take the negative sign in 
(A.5).

Although the temperature perturbation $\overline{\mathrm{T}}^{\star}$ exhibits an algebraic singularity as $\eta \rightarrow \eta_{c}$ (with $\overline{\mathbf{T}}^{*}$ proportional to $\left.\left(\eta-\eta_{c}\right)^{-i}\right)$, nonetheless viscous effects in the $\zeta-O(1)$ layer are sufficient to alleviate this singularity.

This then completes the determination of the critical layer, and resolves the sign ambiguity in (A.5).

\section{Acknowledgements}

A number of the computations were carried out at the University of Manchester Regional Computer Centre with computer time provided under S.E.R.C. Grant. No. GR/E 25702. 


\section{References}

1. Ackerberg, R.C.; and Phillips, J.H.: The Unsteady Laminar Boundary Layer on a Semi-Infinite Flat Plate Due to Small Fluctuations in the Magnitude of the Free-Stream Velocity. J. Fluid Mech., vol. 51, pt. 1, Jan. 11, 1972, pp. 137-158.

2. Brown, S.N.; and Stewartson, K.: On the Propagation of Disturbances in a Laminar Boundary Layer, I. Proc. Cambridge Philos. Soc., vo1. 73, pt. 2, Mar. 1973, pp. 493-503.

3. Brown, S.N.; and Stewartson, K.: On the Propagation of Disturbances in a Laminar Boundary Layer, II. Proc. Cambridge Philos. Soc., vo1. 73, pt. 2, Mar. 1973, pp. 505-514.

4. Duck, P.W.: Laminar Flow Over Unsteady Humps: The Formation of Waves. J. F1uid Mech., vo1. 160, Nov. 1985, pp. 465-498.

5. Duck, P.W.: A Note on a Numerical Method for Treating Time-Periodic Boundary Layers. Submitted to J. Fluid. Mech., 1989.

6. Gibson, W.E.: Unsteady Laminar Boundary Layers. Ph.D. Dissertation, Massachusetts Institute of Technology, 1957.

7. Goldstein, M.E.: The Evolution of Tollmien-Schlichting Waves Near a Leading Edge. J. Fluid Mech., vo1. 127, Feb. 1983, pp. 59-82.

8. Goldstein, M.E.; Sockol, P.M.; and Sanz, J.: The Evolution of Tollmien-Schlichting Waves Near a Leading Edge. Part 2. Numerical Determination of Amplitudes. J. Fluid. Mech., vol. 129, Apr. 1983, pp. 443-454.

9. Holstein, H.: Uber Die Aussere und Innere Reibungsschicht bei Storungen Laminarer Stromungen. Z. Angew. Math. Mech., vo1. 30, 1950, pp. 25-49. 
10. Kestin, J.; Maeder, P.F.; and Wang, H.E.: On Boundary Layers Associated With Oscillating Streams. Appl. Sci. Res., vol. A10, no. $1,1961, \mathrm{pp} .1-22$.

11. Lam, S.H.; and Rott, N.: Theory of Linearized Time-Dependent Boundary Layers. (Avai1. NTIS, AD-246519.) AFOSR-TN-60-1100, 1960.

12. Lees, L.; and Lin, C.C.: Investigation of the Stability of the Laminar Boundary Layer in a Compressible Fluid. NACA TN-1115, 1946.

13. Lighthil1, M.J.: The Response of Laminar Skin Friction and Heat Transfer to Fluctuations in the Stream Velocity. Proc. Roy. Soc. London Series A, vo1. 224, no. 1156, June 9, 1954, pp. 1-23.

14. Lin, C.C.: Motion in the Boundary Layer with a Rapid1y Oscillating External Flow. IX Congress International de Mecanique Appliquee, vol. 4, University of Brussels, 1957, pp. $155-167$.

15. Mack, L.M.: Boundary Layer Linear Stability Theory. Special Course on Stability and Transition of Laminar Flow. AGARD-R-709, AGARD, Paris, France, 1984, pp. 3-1 and 3-81. (Avail. NTIS, $\mathrm{AD}-\mathrm{A} 147243$.

16. Mack, L.M.: Review of Linear Compressible Stability Theory. On Stability of Time Dependent and Spatially Varying Flows, D.L. Dwoyer and M.Y. Hussaini, eds., Springer, 1987, pp. 164-187.

17. Moore, F.K.: Unsteady Laminar Boundary Layer Flow. NACA TN-2471, 1951. 
18. Moore, F.K.: Aerodynamic Effects of Boundary-Layer Unsteadiness. Sixth Anglo-American Aeronautical Conference, J. Bradbrooke and V.A. Libby, eds., Royal Aeronautical Society, Folkestone, 1957, pp. 439-476.

19. Patel, M.H.: On Laminar Boundary Layers in Oscillatory Flow. Proc. Roy. Soc. London Series A, vol. 347, no. 1648, Dec. 9, 1975, pp. 99-124.

20. Pedley, T.J.: Two-Dimensional Boundary Layers in a Free Stream Which Oscillates Without Reversing. J. Fluid. Mech., vol. 55, pt. 2, Sept. 26, 1972, pp. 359-384.

21. Rosenhead, L., ed.: Laminar Boundary Layers. Oxford Univ. Press, 1963.

22. Ryzhov, 0.S.; and Zhuk, V. I.: Internal Waves in the Boundary Layer with the Self-Induced Pressure. J. Mec., vo1. 19, no. 3, 1980, pp. 561-580.

23. Stewartson, K.: On the Impulsive Motion of a Flat Plate in a Viscous Fluid. Q. J. Mech. App1. Math., vol. 4, June 1951, pp. 182-198.

24. Stewartson, K.: The Theory of Laminar Boundary Layers in Compressible Fluids. Oxford University Press, 1964. 


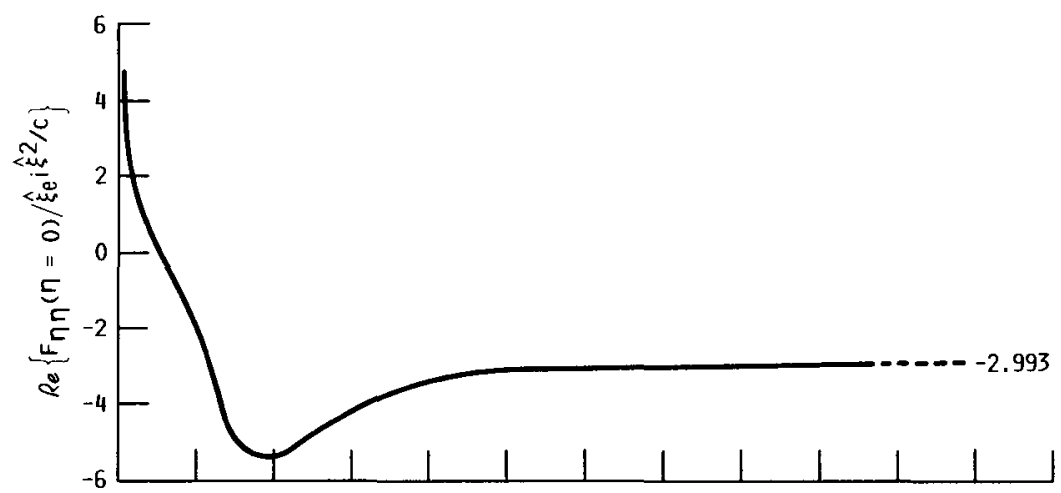

(a)

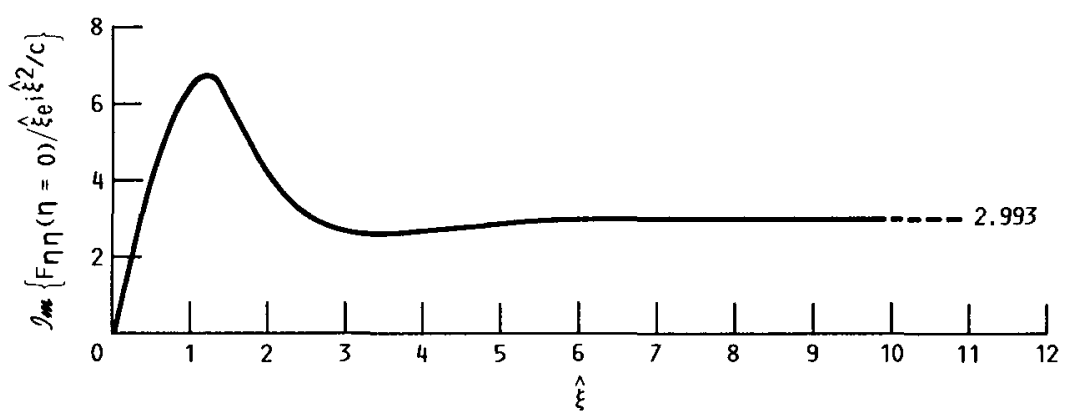

(b)

FIGURE 1. - REAL AND IMAGINARY DISTRIBUTIONS OF $\left\{F n \eta(\eta=0) / \hat{\xi}_{e} i \hat{\xi}^{2} / c\right\}$ FOR $M_{\infty}=\sqrt{2}$ AND $c=1-1 / M_{\infty}$.

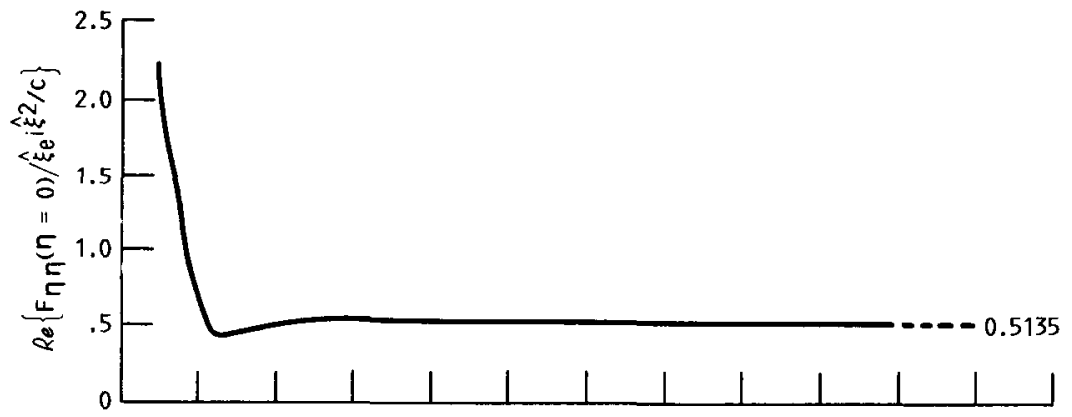

(a)

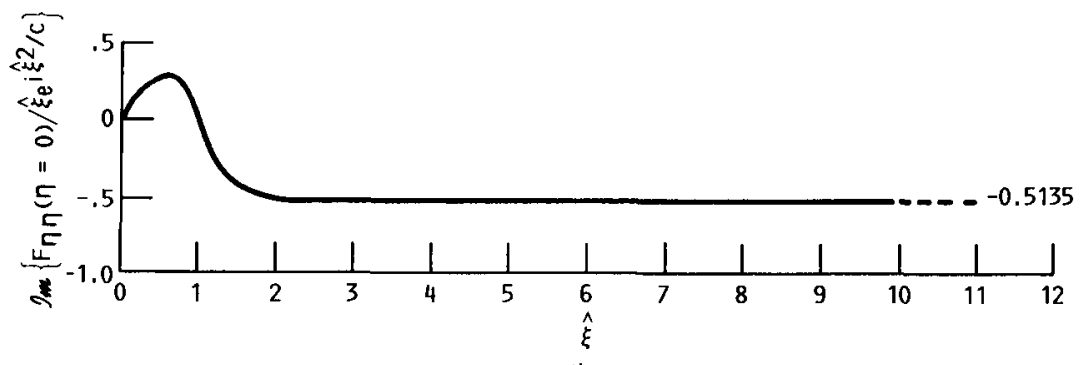

(b)

FIGURE 2. - DETAILS AS FOR FIGURE 1 EXCEPT THAT $c=1+1 / M_{\infty}$. 

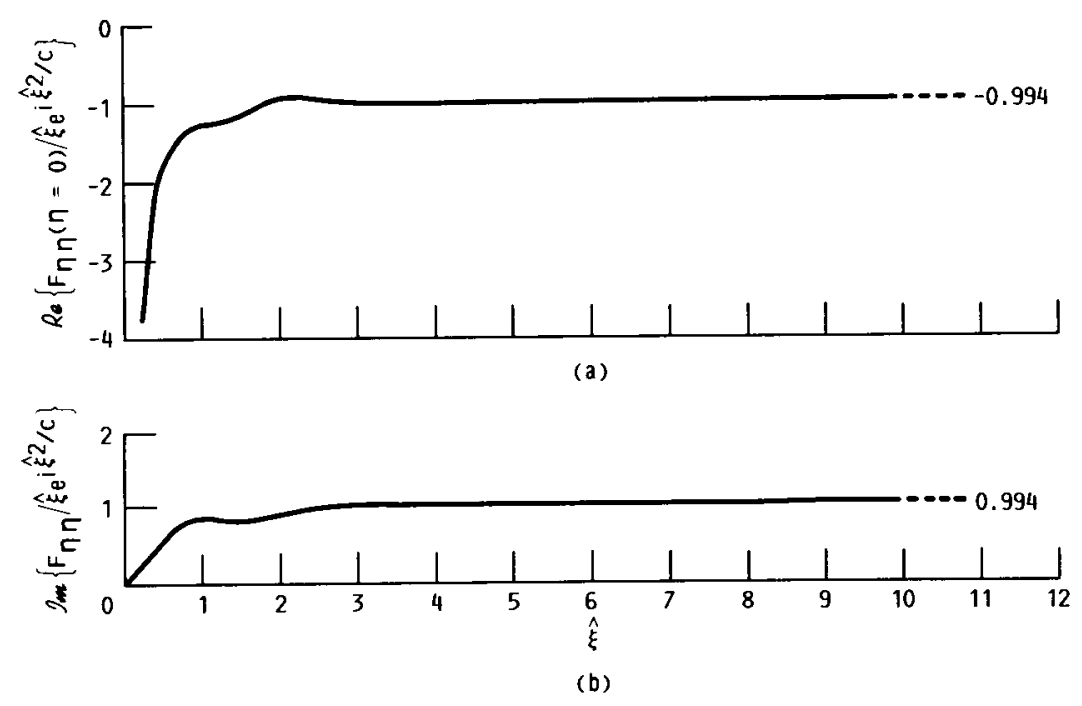

FIGURE 3. - DETAILS AS FOR FIGURE 1 EXCEPT THAT $M_{\infty}=5$.
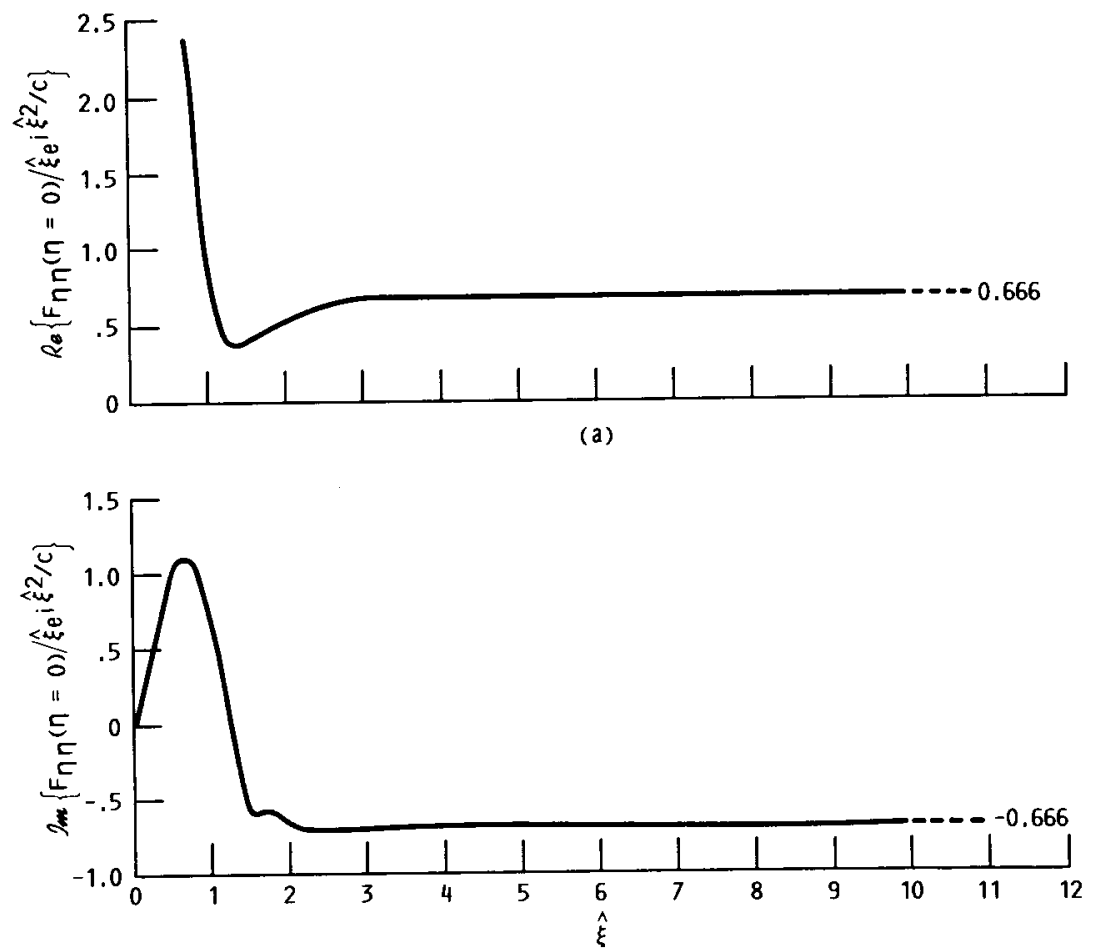

(b)

FIGURE 4. - DETAILS AS FOR FIGURE 2 EXCEPT THAT $\boldsymbol{m}_{\infty}=5$. 

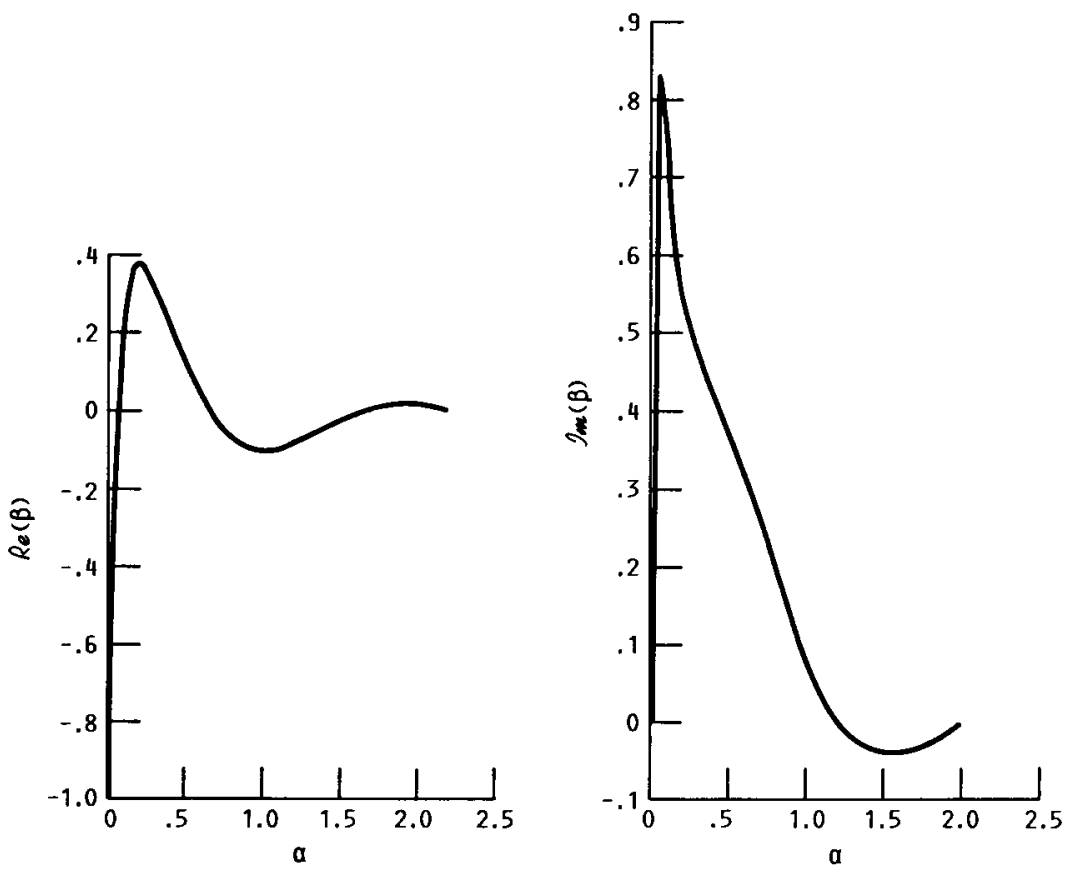

(a)

(b)

FIgURE 5. - REAL AND IMAGINARY DISTRIBUTIONS OF $\beta$ FOR $M_{\infty}=\sqrt{2}$;

$\lambda=(1+i) / 2 ;$ AND $c=1-1 / M_{\infty}$.
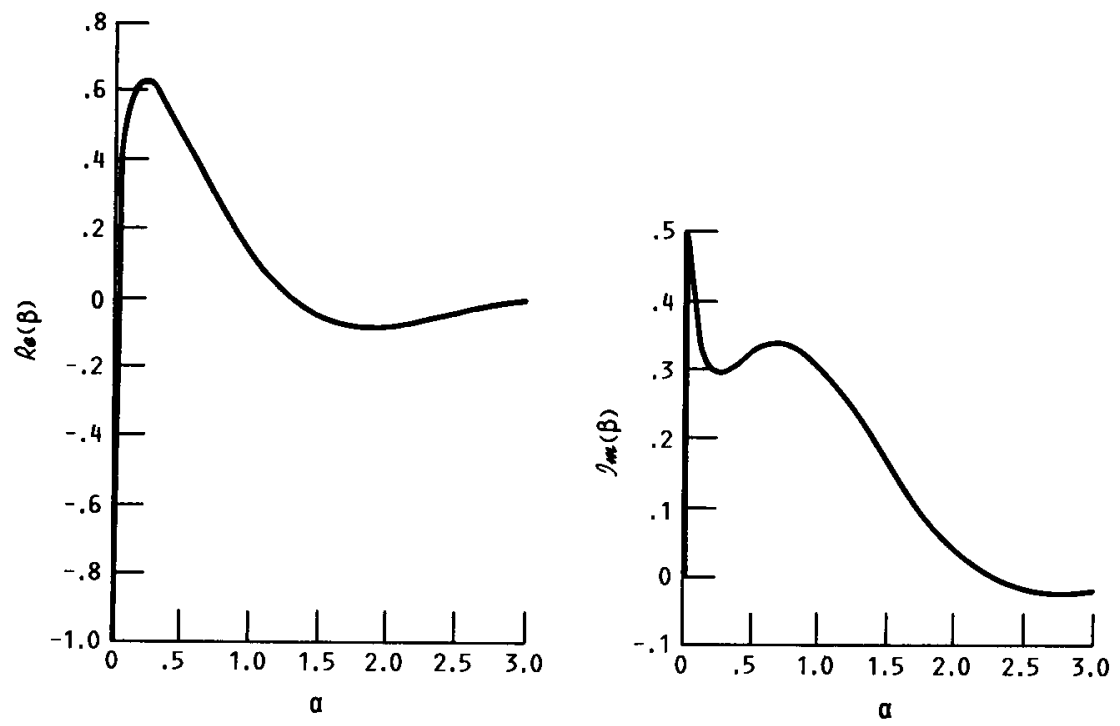

(a)

(b)

FIGURE 6. - REAL AND IMAGINARY DISTRIBUTIONS OF $\beta$ FOR $\boldsymbol{M}_{\infty}=\sqrt{2}$; $\lambda=(1+i) / 4 ; c=1-1 / M_{\infty}$. 


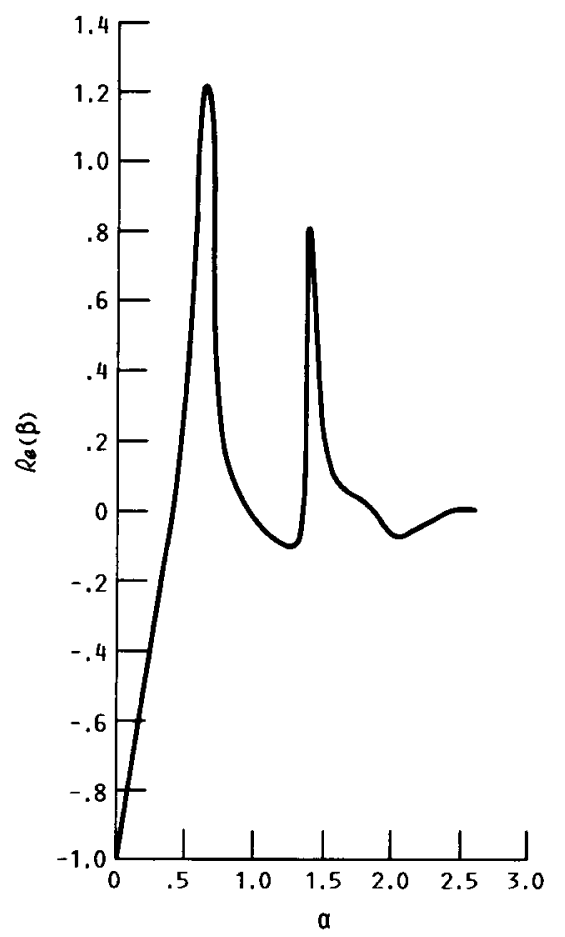

(a)

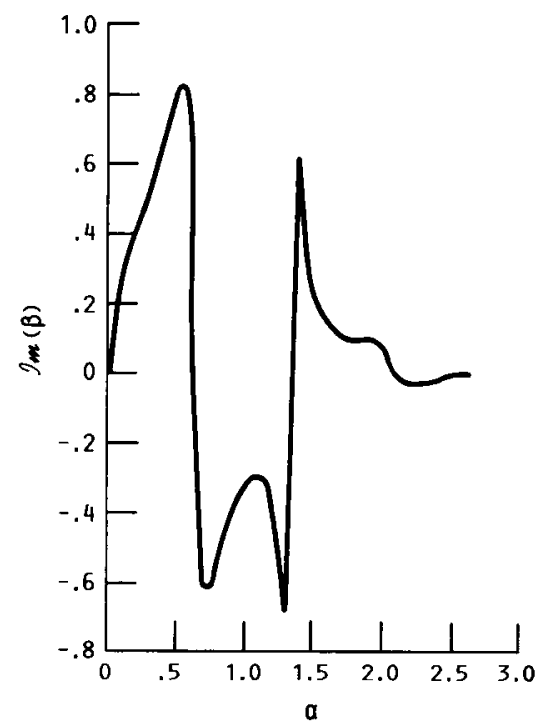

(b)

FIGURE 7. - DETAILS AS FOR FIGURE 5 EXCEPT THAT $c=1+1 / M_{\infty}$.

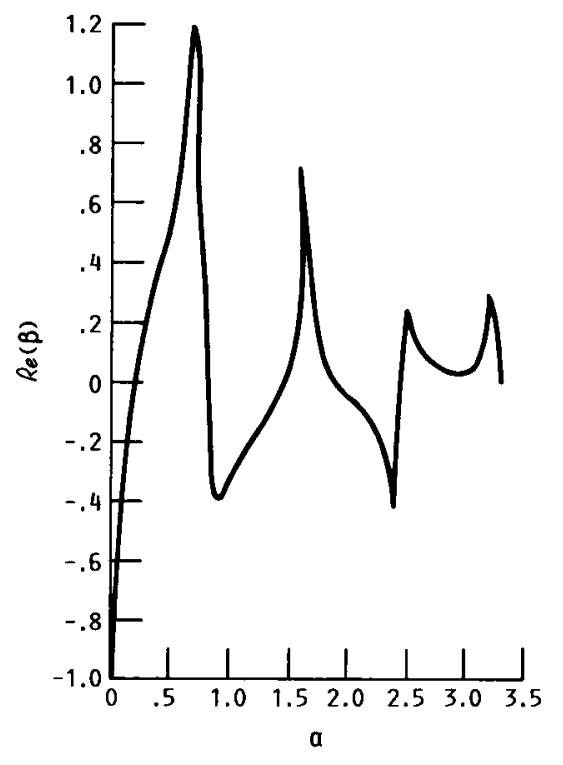

(a)

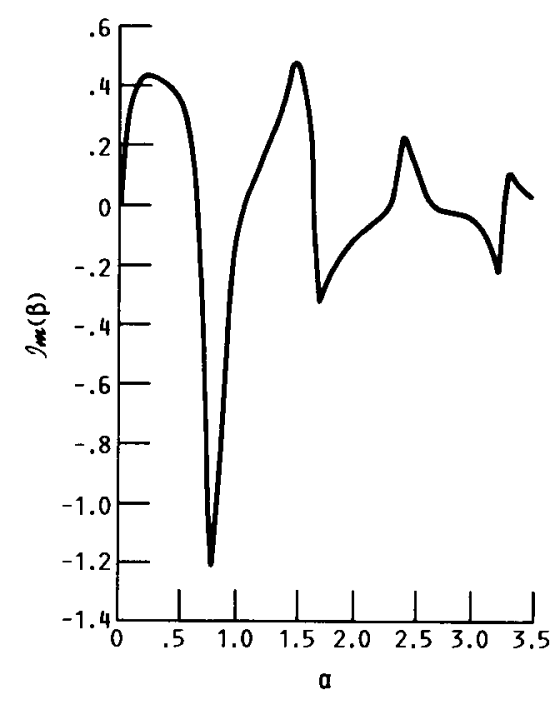

(b)

FIGURE 8. - DETAILS AS FOR FIGURE 6 EXCEPT THAT $c=1+1 / M_{\infty}$. 


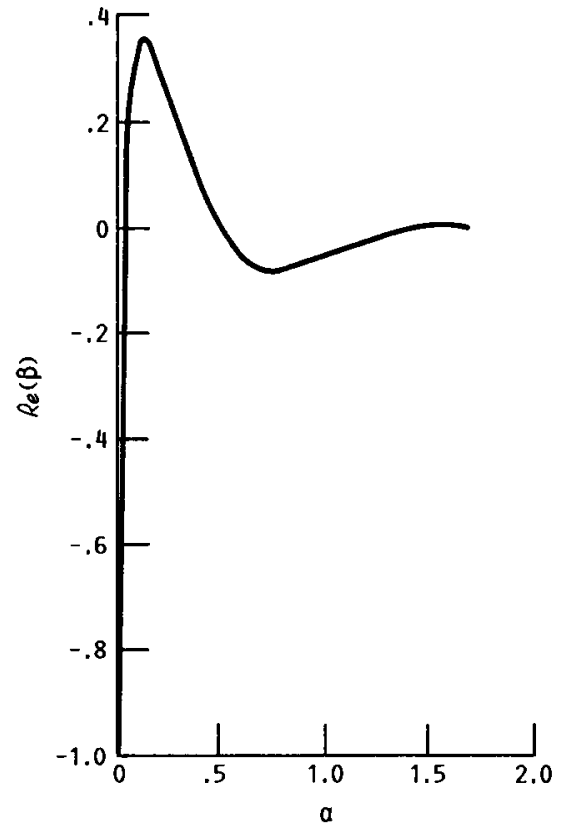

(a)

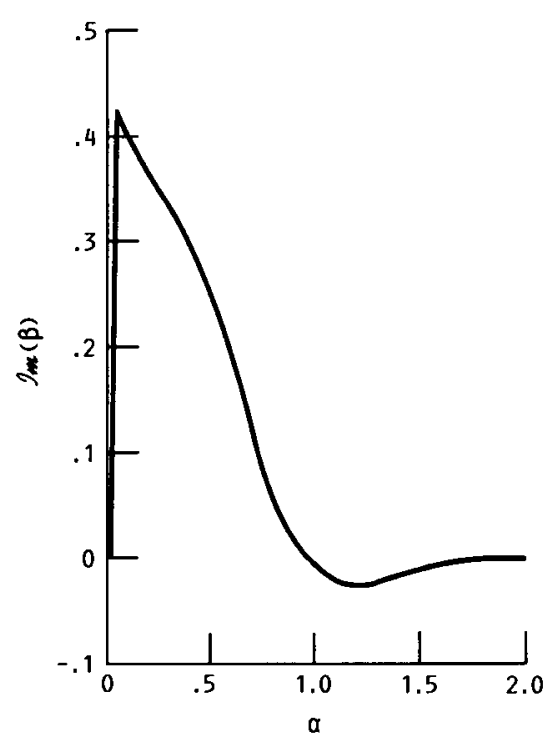

(b)

FIGURE 9. - REAL AND IMAGINARY DISTRIBUTIONS OF $\beta$ FOR $M_{\infty}=5 ; \lambda=(1+i) / 2$; $c=1-1 / M_{\infty}$.

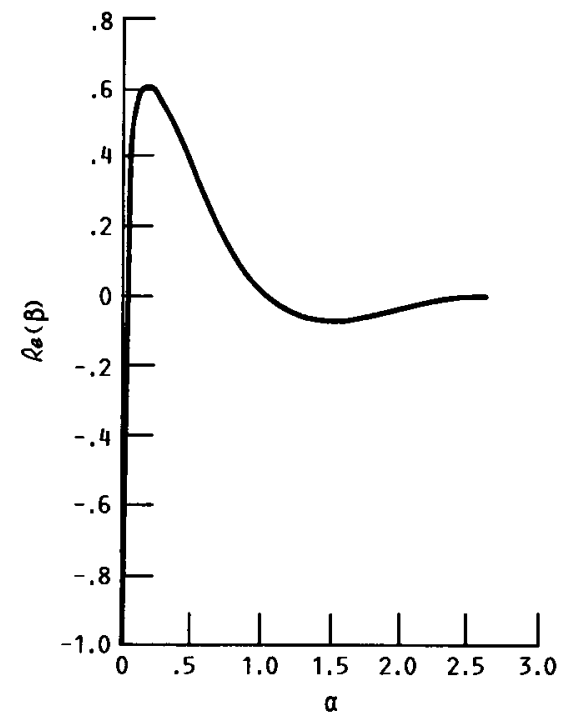

(a)

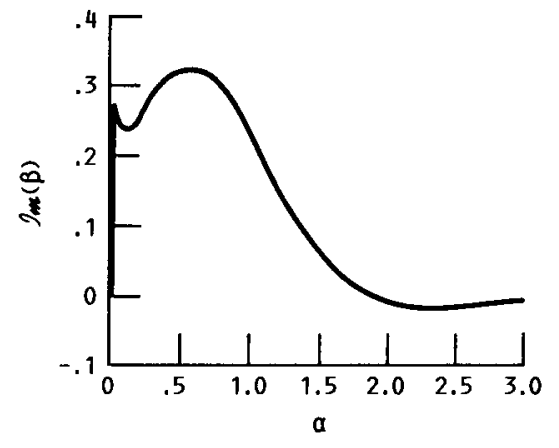

(b)

FIGURE 10. - DETAILS AS FOR FIGURE 9 EXCEPT THAT $\lambda=(1+i) / 4$. 


\section{Report Documentation Page}

\begin{tabular}{ll|l} 
1. Report No. & $\begin{array}{l}\text { NASA TM-101965 } \\
\text { ICOMP-89-6 }\end{array}$ & 2. Government Accession No. \\
\hline
\end{tabular}

4. Title and Subtitle

The Response of a Laminar Boundary Layer in Supersonic Flow to Small Amplitude Progressive Waves

3. Recipient's Catalog No.

5. Report Date

March 1989

6. Performing Organization Code

8. Performing Organization Report No.

E-4663

Peter W. Duck

10. Work Unit No.

$505-62-27$

9. Performing Organization Name and Address

National Aeronautics and Space Administration

Lewis Research Center

Cleveland, Ohio 44135-3191

12. Sponsoring Agency Name and Address

National Aeronautics and Space Administration

Washington, D.C. 20546-0001

15. Supplementary Notes

Peter W. Duck, Institute for Computational Mechanics in Propulsion, Lewis Research Center (work funded under Space Act Agreement C99066G); presently at the Department of Mathematics, University of Manchester, Manchester England.

16. Abstract

In this paper the effect of a small amplitude progressive wave on the laminar boundary layer on a semi-infinite flat plate, due to a uniform supersonic freestream flow, is considered. The perturbation to the flow divides into two streamwise zones. In the first, relatively close to the leading edge of the plate, on a transverse scale comparable to the boundary layer thickness, the perturbation flow is described by a form of the unsteady linearized compressible boundary layer equations. In the freestream, this component of flow is governed by the wave equation, the solution of which provides the outer velocity conditions for the boundary layer. This system is solved numerically, and also the asymptotic structure in the far downstream limit is studied. This reveals a breakdown and a subsequent second streamwise zone, where the flow disturbance is predominantly inviscid. The two zones are shown to match in a proper asymptotic sense.
17. Key Words (Suggested by Author(s))

Unsteady boundary layers

Receptivity

Supersonic flow
18. Distribution Statement

Unclassified - Unlimited

Subject Category 34

19 Security Classif. (of this report)

20. Security Classif. (of this page)

21. No of pages

22. Price*

Unclassified

Unclassified

38

$\mathrm{A03}$ 